\title{
Application of ultrasound for muscle assessment in sarcopenia: towards standardized measurements
}

\author{
Stany Perkisas ${ }^{1,18}$ - Stéphane Baudry ${ }^{2,18}$. Jürgen Bauer ${ }^{3} \cdot$ David Beckwée $^{4,5} \cdot$ Anne-Marie De Cock ${ }^{1}$. \\ Hans Hobbelen ${ }^{6,19} \cdot$ Harriët Jager-Wittenaar ${ }^{6,20}$. Agnieszka Kasiukiewicz ${ }^{7}$. Francesco Landi ${ }^{8}$. Ester Marco ${ }^{9,10,11}$. \\ Ana Merello $^{12} \cdot$ Karolina Piotrowicz $^{13} \cdot$ Elisabet Sanchez $^{12} \cdot$ Dolores Sanchez-Rodriguez $^{11,14,15,16}$. \\ Aldo Scafoglieri ${ }^{17,18} \cdot$ Alfonso Cruz-Jentoft $^{12} \cdot$ Maurits Vandewoude $^{1,18}$
}

Received: 15 June 2018 / Accepted: 29 August 2018

(c) European Geriatric Medicine Society 2018

\begin{abstract}
Purpose Measurement of muscle mass is paramount in the screening and diagnosis of sarcopenia. Besides muscle quantity however, also quality assessment is important. Ultrasonography (US) has the advantage over dual-energy X-ray absorptiometry (DEXA) and bio-impedance analysis (BIA) to give both quantitative and qualitative information on muscle. However, before its use in clinical practice, several methodological aspects still need to be addressed. Both standardization in measurement techniques and the availability of reference values are currently lacking. This review aims to provide an evidence-based standardization of assessing appendicular muscle with the use of US.

Methods A systematic review was performed for ultrasonography to assess muscle in older people. Pubmed, SCOPUS and Web of Sciences were searched. All articles regarding the use of US in assessing appendicular muscle were used. Description of US-specific parameters and localization of the measurement were retrieved.

Results Through this process, five items of muscle assessment were identified in the evaluated articles: thickness, crosssectional area, echogenicity, fascicle length and pennation angle. Different techniques for measurement and location of measurement used were noted, as also the different muscles in which this was evaluated. Then, a translation for a clinical setting in a standardized way was proposed.

Conclusions The results of this review provide thus an evidence base for an ultrasound protocol in the assessment of skeletal muscle. This standardization of measurements is the first step in creating conditions to further test the applicability of US for use on a large scale as a routine assessment and follow-up tool for appendicular muscle.
\end{abstract}

Keywords Sarcopenia $\cdot$ Ultrasound $\cdot$ Muscle assessment

\section{Introduction}

Sarcopenia is becoming one of the biggest health care challenges that arises together with increasing age expectations [1]. It is associated with mortality, functional decline,

The authors are part of the SARCUS working group on behalf of the Sarcopenia Special Interest Group of the European Geriatric Medicine Society.

Electronic supplementary material The online version of this article (https://doi.org/10.1007/s41999-018-0104-9) contains supplementary material, which is available to authorized users.

Stany Perkisas

Stany.perkisas@zna.be

Extended author information available on the last page of the article disability, a higher rate of falls, a higher incidence of hospitalizations, increased health care costs and a lower quality of life [2]. Therefore, clear cutoffs are desirable for an early diagnosis. However, there is still some debate about a universal definition. The currently most used operational definition is the age-related decline of muscle mass, together with the decline of strength and/or function [3]. This definition can still be regarded as largely heterogeneous. This debate is continued in the use of specific cutoff points for muscle mass, as is clearly seen in the many criteria that are proposed by various international organizations [3-8].

When applying these criteria, it is evident that in diagnosing sarcopenia, muscle mass still holds the most weight, more than strength or function. This also finds its way in a case finding algorithm, published by the European Working Group for Sarcopenia in Older People [3], where there 
can be no diagnosis of sarcopenia without the assessment of muscle mass. This focus on muscle mass, however, gives way to a few practical implications, as there seems to be a large cleft between guidelines and clinical practice.

First of all, the prevalence of sarcopenia is highly dependent on the diagnostic method used [9]. As stated, there are many different cutoff points for diagnosing sarcopenia through muscle mass. All are either based on dual-energy X-ray absorptiometry (DXA) or bio-impedancemetry (BIA); currently, there are no cutoff values for either CT or MRI. DXA cutoff points are based on skeletal muscle mass index (SMI), which is the sum of the all the appendicular muscle mass, divided by height. For DXA SMI, three groups of cutoff points are used [10-12]. BIA also uses SMI, with one cutoff point proposition based on BIA-predicted skeletal muscle mass [13] and the other based on absolute muscle mass $[14,15]$. It is unclear in how far both DXA and BIA are available in clinical practice.

Secondly, it has become clear that measuring muscle quantity, i.e., muscle mass, in itself is only part of the problem of age-related muscle degradation. Also the muscle architectural qualities - here used synonymously as muscle quality —need to be assessed, as volume in itself has no linear relation with either strength or function. Muscle quality is a broad term and is in current literature used in two meanings. On one hand, it can mean the muscle strength or muscle power per unit of muscle mass [16-18]. On the other hand-as in this study - it can be used as description of the components of the muscle described [19]. The neural component in the process of sarcopenia could possibly amount up to 50\% [19], but it is clear that shear volume alone does not explain the rest. Measuring the components of this volume is vital $[20,21]$. The assessment of these components cannot be done either by DXA or BIA [22], although there is some evidence that multi-frequency bioimpedance and segmental bioelectrical impedance spectroscopy could measure muscle composition to a certain degree and give information regarding skeletal muscle physiology [23, 24]. For assessing muscle quality, computerized tomography (CT) and magnetic resonance imaging (MRI) are considered 'gold standards' [25], in as far this is possible, because many different scanning techniques exist: CT muscle attenuation [26], diffusion tensor MRI [27], Dixon MRI [28], proton magnetic resonance spectroscopy (MRS) [29], 13C-MRS [30] and 31P-MRS [31]. For both CT and MRI, whole body scanning can be done, as also single/multiple slice scanning [32-34]. The former are considered as superior, but adequate data are present to use the latter as a convenient alternative [3]. In clinical practice, however, CT and MRI are not practical, due to the limited availability, lack of portability, high cost and radiation exposure (CT). Also, currently there are no cutoff values for either CT or MRI. This way, current guidelines and criteria fall short, in the way that they are often not applicable in a clinical routine setting due to practical reasons as stated above, and do not yet have incorporated the quality aspect of muscle mass. There is an important need for an instrument that can give information about both muscle quantity and quality, that is cheap and easily available in routine practice, and that can be used in large populationbased screenings.

Ultrasonography or ultrasound (US) could fill this gap.

US is a well-studied technique that has already proven its worth in the detection of neuromuscular pathology with positive predictive values of up to $90 \%$ [35] and in the assessment of muscle-tendon interactions [36]. US is a portable, inexpensive, non-invasive technique without using ionising radiation that also has a high repeatability. Regarding muscle mass assessment, US-based measurements have a strong positive correlation with DEXA [37-40], CT [41] and MRI-based measurements [42-44]. For the estimation of muscle quality and quantity, US is a valid and accurate technique $[45,46]$. The validity of ultrasound to discern architectural properties has also been demonstrated in cadaver validation studies [47-51]. It has good intra- and interrater reliability [45], as well as test-retest reliability [52], both in an elderly people [45] as in a younger population [53]. However, it is unclear which anatomical site is best to be used for specific outcomes, e.g., prediction of total skeletal muscle mass [54]. It must be taken into account that not all peripheral muscles decline alike [55, 56]. In this regard, prediction equations for total skeletal muscle mass need further validation [45]. Also, there are hardly any reference data currently available, as there is no standardization of the measurement technique. To our knowledge, there are only four studies giving limited reference data, for muscle thickness and echo intensity of selected muscles [57-60].

This review aims to provide a standardization of ultrasound measurements for assessing muscle mass in the assessment of sarcopenia. To the best of our knowledge, there are no hard clinical data to prefer assessing one muscle or set of muscles over another, or to choose one type of measurement and disregards the others. Therefore, an overview will be given of how appendicular muscles are measured in the literature so far, followed by a proposition of how to do a muscle assessment in a standardized way. These propositions will be based on the literature and consensus within the review group. Among these propositions, there will be a list summarizing which information needs to be minimally included in a protocol when planning future studies. This way, we hope to advance the study of the application of ultrasound in sarcopenia assessment in different settings [61, 62]. 


\section{Methods}

\section{Registration}

The protocol for this systematic review has been registered at PROSPERO (Registration number CRD42018085587).

\section{Search strategy}

The search strategy was set up based on three main components: elderly (population) [63], ultrasound (exposure), and muscle (outcome). For this, a modified PECO model for clinical questions was used. The search was performed in Pubmed, Web of Science and Cochrane Library, up until the 20th of January 2018. All eligible studies in English, German, French and Dutch were screened for their applicability. Studies regarding the use of ultrasound in the assessment of muscle mass were considered for this review. Bibliographic lists of included papers were hand-searched for additional studies. Animal studies, studies using cadaver specimens, studies assessing non-appendicular muscle, case reports, letters to the editor, editorials and (systematic) reviews were excluded.

Search structure for PubMed was as follows: ((((Elderly[tiab] OR community-dwelling[tiab] OR geriatric[tiab] OR Frailty[tiab] OR Ageing[tiab] OR elders[tiab] OR Frail[tiab] OR "postmenopausal women"[tiab] OR aging[tiab] OR older[tiab] OR residents[tiab] OR "old people"[tiab] OR nursing homes[mh] OR aging[mh] OR frail elderly[mh] OR homes for the aged[mh] OR aged, 80 and over[mh])))) AND ((()((()(Ultrasonography[Mesh]) OR Ultrasound) OR Echograph*) OR Ultrasonograph*) OR Ultrasonic) OR Echotomograph*) OR Sonograph*)) AND ((“Muscles”[Mesh] OR "Lean tissue" OR "Lean mass" OR "Lean body mass" OR muscle OR "Fat free mass"))).

Search structure for SCOPUS was as follows: ((TITLEABS-KEY (elder*)) OR (TITLE-ABS-KEY (communitydwelling)) OR (TITLE-ABS-KEY (geriatric)) OR (TITLEABSKEY (frail*)) OR (TITLE-ABS-KEY (ag*ing)) OR (TITLE-ABSKEY ("postmenopausal women")) OR (TITLE-ABS-KEY (old*)) OR (TITLE-ABSKEY (resident*))) OR (TITLE-ABS-KEY ("nursing homes"))) AND ((TITLE-ABSKEY (ultrasound)) OR (TITLE-ABS-KEY (echograph*)) OR (TITLE-ABSKEY (ultrasonograph*)) OR (TITLE-ABS-KEY (ultrasonic)) OR (TITLE-ABSKEY (echotomograph*)) OR (TITLE-ABS-KEY (sonograph*))) AND ((TITLE-ABSKEY (muscle*)) OR (TITLE-ABS-KEY ("Lean tissue")) OR (TITLE-ABS-KEY ("Lean mass")) OR (TITLE-ABS-KEY ("Lean body mass")) OR (TITLE-ABSKEY ("Fat free mass"))).
Search structure for Web of Science was as follows: Elder* OR community-dwelling OR geriatric OR Frail* OR Ageing OR Frail OR "postmenopausal women" OR old* OR resident* OR "nursing homes". Ultrasound OR Echograph* OR Ultrasonograph* OR Ultrasonic OR Echotomograph* OR Sonograph*. Muscle* OR "Lean tissue" OR "Lean mass" OR "Lean body mass" OR "Fat free mass".

An overview of the study selection process is shown in Fig. 1. After deleting duplicates, abstracts were gathered. Abstracts were divided among 12 independent reviewers, experienced in either geriatrics, physical therapy, radiology or body composition. One other reviewer (SP) screened all the abstracts. Title and abstract of all manuscripts were screened for eligibility, reviewers being blinded from each other, using the Rayyan web-based software [64]. Disagreements were resolved by consensus within the group. All review articles, case reports, letters to the editor, and editorials were excluded. Then, the selected articles were used for full-text reading. Again, the full-text articles were divided among the same 12 independent reviewers as in the previous step. However, for the reviewers, the full texts to be reviewed were not the same as the abstracts that were reviewed. In other words, each reviewer reviewed two different sets of publications. Reasons for exclusion of an article after full-text assessment were: absence of clear description of location of measuring point, absence of clear description of muscle measured, content being article outside the scope of the manuscript, or referencing to another article regarding measurement technique/location. For the last category, the article to which was referred for measurement technique/ location, was checked for inclusion. If not already included, it was added.

\section{Results}

\section{Search strategy}

The initial search yielded 17.579 abstracts (PubMed $=5565$, SCOPUS $=7255$, Web of Science $=4759$ ). There was one additional record identified through other sources. After deleting duplicates $(n=2751), 14.829$ abstracts were screened. Of these, 359 articles were withheld. After fulltext assessment, 76 articles were used in this review. See also Fig. 1 for details about the study selection process.

All articles included in this paper are detailed in a supplemental volume (Table S1), with cohort size, age (median or range) and ethnicity.

Mean cohort size was 58 (median 44, range 9-347). One study did not mention the cohort size. Two papers did not have cohorts as they were suggestions for US protocols. 


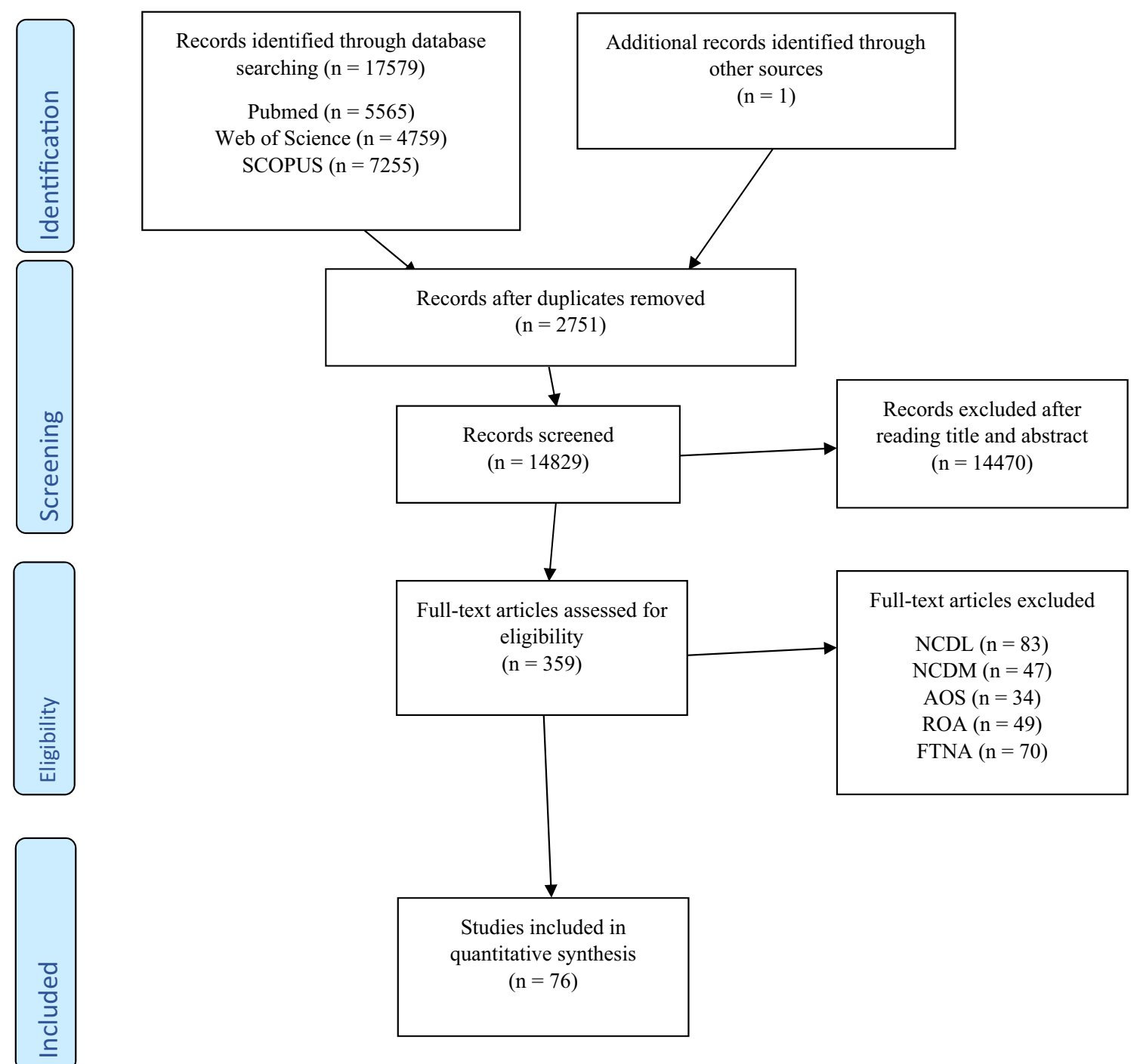

Fig. 1 Overview of the study selection process using the PRISMA 2009 flow chart [166]. NCDL no clear description of location of measuring point, $N C D M$ no clear description of muscle measured,

Age was clearly described in 38 studies. In 33 studies, only ranges were given. Three studies did not mention age. In the two protocol studies, no patients were used so no age was noted.

Ethnicity was very poorly described explicitly. Eight studies mentioned Asian ethnicity (one Chinese, seven Japanese), one study mentioned Caucasian ethnicity, and one study mentioned white/Indian ethnicity. One study mentioned different ethnicities: Caucasian 87\%, Asian 29\%, native American 3\%, African 1\%. In two studies (protocol), ethnicity was not applicable.

The sex of study subjects was not included in this review.

As there is no gold standard in US muscle assessment, it was difficult to have a good quality assessment of the
$A O S$ article outside of scope of manuscript, $R O A$ referencing to other articles for measurement technique, FTNA full-text not available

included articles. Although a multitude of research questions were present, it must be noted that no studies compared different US techniques in the assessment of muscle.

\section{Patient positioning pre-investigation}

Only seven studies stated clearly which subject side was assessed. In five studies, the dominant side was used [65-69]. Only one study specified using the non-dominant side [70]. One study used only the right side, not mentioning if this was the dominant side or not [71].

Regarding the state of the muscle assessed, one article mentioned letting the test subject perform three maximum contractions of the muscle assessed before measurement 
European Geriatric Medicine

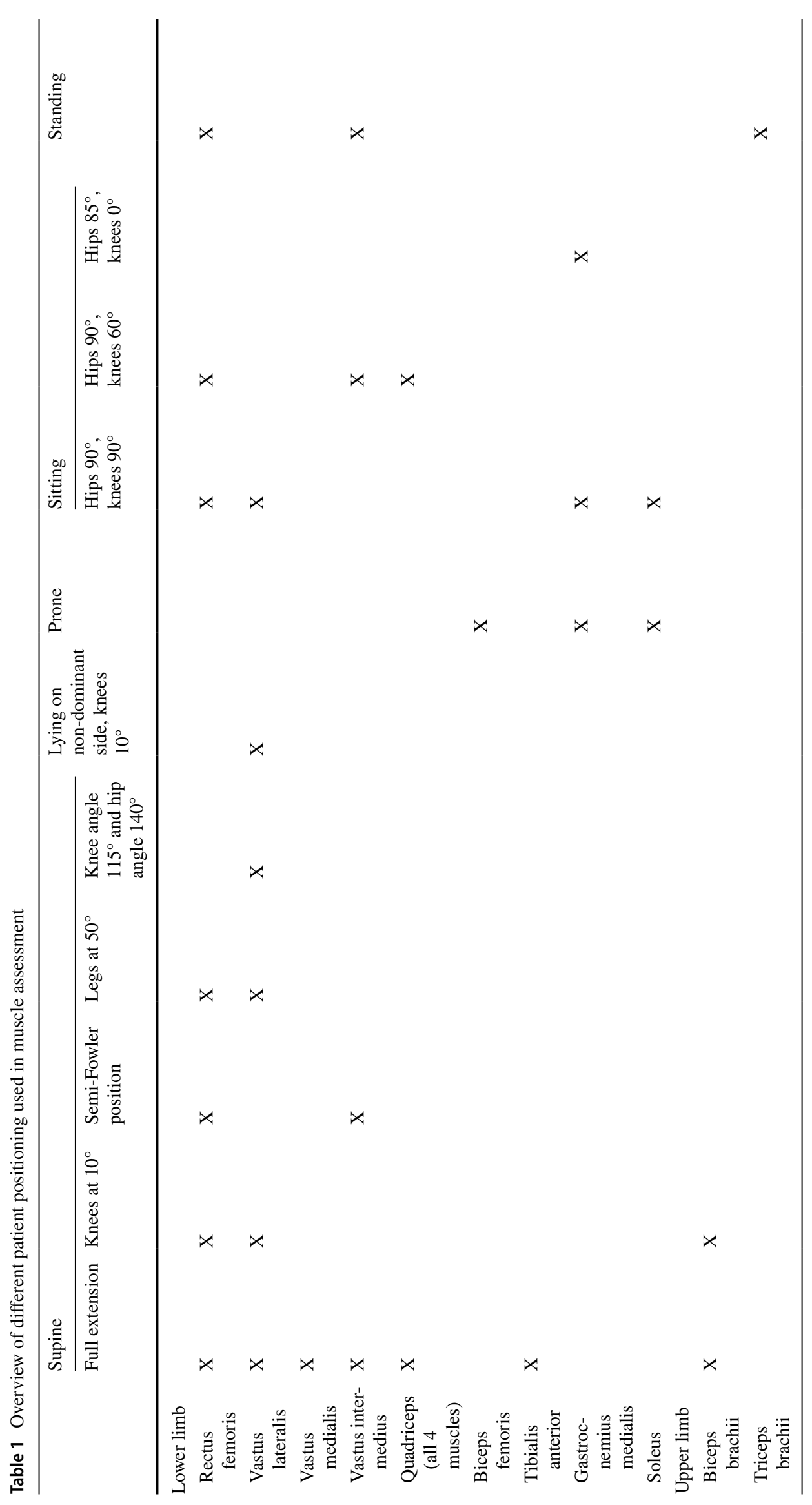

Springer 
[65]. Two studies had the test subjects being relaxed for $15 \mathrm{~min}$, one to allow fluid shifts to stabilize [72], the other to avoid muscle contraction-induced fluid shifts and muscle blood flow during the measurements [73]. The latter, therefore, also specified to have done all ultrasound measurement before any functional testing. Two groups refrained the study subjects from rigorous exercises, one only for the same day [74], one for the last $48 \mathrm{~h} \mathrm{[75].}$

The position of the study subject was dependent on the specific muscle investigated, but also herein there were some differences. For an overview of the different positions used in selected muscles, see Table 1 . In the supplemental material, a table is included that indicates which study assessed which muscle at a certain position (Table S2).

For supine and prone positions, study subject is regarded as having hips and shoulders in neutral position, knees and elbows in full extension, ankles at $90^{\circ}$, unless specified otherwise. It has to be mentioned that it is important to correctly describe the positioning, as the angle of joints is not standardized in one direction, e.g., knees in $150^{\circ}$ or $30^{\circ}$ could mean the same. In none of the studies, it was described whether the supine position included the feet being of the table or not. One study, assessing the gastrocnemius medialis, specified having the tibiotalar joint angle at $115^{\circ}$, for which they had a cast specially made for all participants [69].

\section{Upper leg muscles}

- Rectus femoris was assessed in supine position in full extension [65, 68, 72, 76-86], with knees at $10^{\circ}$ [87-89], in semi-Fowler position [90-92]. It was assessed in sitting position with hips and knees at $90^{\circ}[66,93]$ and with hips at $90^{\circ}$ and knees at $60^{\circ}$ [94]. Lastly, it was also assessed standing [95, 96].

- Vastus lateralis was assessed in supine position in full extension [72, 78, 84, 85, 89, 97-101], with knees at $10^{\circ}$ [88], with legs at $50^{\circ}$ [71] and with knee angle at $115^{\circ}$ and hip angle at $140^{\circ}$ [102]. It was assessed lying in the non-dominant side with knees at $10^{\circ}$ lateralis [87]. Lastly, it was assessed sitting with hips and knees at $90^{\circ}$ [103].

- Vastus medialis was assessed in supine position in full extension [72, 84, 104].

- Vastus intermedius was assessed in supine position in full extension [72, 76-79, 84] and in semi-Fowler position [90]. It was assessed in sitting position with hips at $90^{\circ}$ and knees at $60^{\circ}$ [94]. Lastly, it was also assessed standing [96].

- Quadriceps-comprising all 4 muscles: rectus femoris, vastus medialis, vastus intermedius and vastus lateraliswere assessed in supine position in full extension [105].
It was also assessed in sitting position with hips at $90^{\circ}$ and knees at $60^{\circ}$ [106].

- Biceps femoris was only assessed in prone position [78].

\section{Lower leg muscles}

- Tibialis anterior was only assessed in full extension [97].

- Gastrocnemius medialis was assessed in prone position [78, 82, 97, 102, 107-111] and in sitting position with hips and knees at $90^{\circ}[69,103,112]$.

- Soleus was assessed in prone position [78] and in sitting position with hips and knees at $90^{\circ}$ [112].

\section{Upper arm muscles}

- Biceps brachii was only assessed in full extension [57, 97, 113].

- Triceps brachii was assessed standing [114, 115]. One study that assessed triceps brachii did not mention the patient position [116].

\section{System and system settings}

Many different types of ultrasound machines were used, from various manufacturers (SonoSite, GE Healthcare, Siemens, Mindray, Philips, Toshiba, Hitachi Aloka Medical, Esaote, Fukuda Denshi, Hewlett-Packard, Telemed). A summary of the different types used is beyond the scope of this article.

Every research group used B-mode ultrasound, and all but one used a linear transducer probe. The only article using a curved transducer had as preposition the validation of the use of a curved versus a linear transducer [91].

The length of the transducer varied from 3.8 to $7 \mathrm{~cm}$. The frequency used was described in 63 cases, and ranged from 3 to $15 \mathrm{MHz}$.

In 13 cases, the bandwidth instead of the exact frequency was described: $10-15 \mathrm{MHz}(n=1), 7-12 \mathrm{MHz}(n=3)$, 6-13 MHz $(n=1), 5-12 \mathrm{MHz}(n=2), 5-10 \mathrm{MHz}(n=3)$, 3-13 MHz $(n=1), 3-12 \mathrm{MHz}(n=1), 3-11 \mathrm{MHz}(n=1)$.

In 50 articles, a clear frequency was described. The most common used frequency was $7.5 \mathrm{mHz}(n=23)$; further frequencies used were $15 \mathrm{MHz}(n=1), 13.6 \mathrm{MHz}(n=1)$, $12 \mathrm{MHz}(n=7), 10 \mathrm{MHz}(n=6), 9 \mathrm{MHz}(n=3), 8 \mathrm{MHz}$ $(n=7), 5 \mathrm{MHz}$ (linear array, $n=6), 5 \mathrm{MHz}$ (curved array, $n=1)$.

No information on the inclination of the probe was noted.

Some additional system settings were described. Most were on image depth (focus point) and general gain.

Image depth was described in 7 cases: $45 \mathrm{~mm}(\mathrm{~mm})$ [71], $50 \mathrm{~mm}$ [87, 88, 117], $60 \mathrm{~mm}[101,118]$ and $70 \mathrm{~mm}$ [72]. 
General gain (in decibels, $\mathrm{dB}$ ) was described in 15 cases: was either set to $50 \mathrm{~dB}[82,83,87,117,119], 58 \mathrm{~dB}$ [120], $68 \mathrm{~dB}$ [71, 108, 111, 121], $85 \mathrm{~dB}$ [94], $86 \mathrm{~dB}$ [122] or $90 \mathrm{~dB}$ $[72,84,123]$.

Time gain compensation was described as being in a neutral position in two cases $[72,124]$.

Dynamic range was described in five cases: being set to 40 [121], set to 69 [120], or set to 72 [83, 117, 119].

Compression-which is altering the display of the range of echo intensities to end up with a lower amount of different shades of gray-was described in one study as set to $70 \mathrm{~dB}$ [122].

No further system settings were described.

For image post-processing and measurement, 35 of the 75 articles mentioned the use of additional software. These included Image J [125] ( $n=26)$, Matlab (The MathWorks, Inc., Natick, Massachusetts, United States) $(n=2)$ and Photoshop (Adobe, Adobe Systems Incorporated, San Jose, California, United States) $(n=7)$.

\section{Components and measuring points}

Five main components were distilled from the literature: muscle thickness (MT), pennation angle (PA), fascicle length (Lf), echo intensity (EI) and cross-sectional area (CSA).

Four studies mentioned that all measurements were done three times [66, 67, 91, 97], and after that, the mean value was taken. Two other studies mentioned doing all measurements five times $[89,97]$. One of the latter two discarded the highest and lowest value, and then took the mean value of the remaining three [89].

Muscle thickness was defined as the distance between deep and superficial aponeurosis [97, 109]. It can be expressed either in centimetres $(\mathrm{cm})$ or in millimetres $(\mathrm{mm})$.

Cross-sectional area was divided into two: anatomical cross-sectional area (ACSA) and physiological cross-sectional area (PCSA). Anatomical cross-sectional area was defined as the area of cross-section of a muscle perpendicular to its longitudinal axis. Physiological cross-sectional area was defined as the area of cross-section of a muscle perpendicular to its fibers. In non-pennate muscle, ACSA and PCSA are the same; in pennate muscles they are not. ACSA underestimates the number of total fibers in a pennated muscle. Muscle strength is more correlated with PCSA than with ACSA because the former represents the maximal number of acto-myosin crossbridges that can be activated in parallel during contraction [126]. Therefore, when studying muscle strength, it is not advised to only measure ACSA.

Table 2 Measuring points and components of quadriceps muscles used in assessment

\begin{tabular}{|c|c|c|c|c|c|c|c|c|c|}
\hline Proximal point & Distal point & Distance & MT & CSA & ACSA & PCSA & FL & PA & EI \\
\hline \multirow[t]{6}{*}{ Greater trochanter } & Popliteal crease & $60-70 \%$ & $\mathrm{RF}$ & & & & & & \\
\hline & Lateral condyle & $30 \%$ & VM, VL & RF & & & VL & VM, VL & VM, VL \\
\hline & & $50 \%$ & RF, VL, VI & RF, VL & VL & & VL & RF, VL, VI & RF, VL, VI \\
\hline & & $2 / 3$ & RF, VL & VL & & & VL & VL & VL \\
\hline & Articular cleft of knee & $50 \%$ & RF, VI & & & & & & \\
\hline & $\begin{array}{l}\text { Proximal border of } \\
\text { patella }\end{array}$ & $50 \%$ & & & $\mathrm{RF}$ & $\mathrm{RF}$ & & & \\
\hline \multirow{8}{*}{$\begin{array}{l}\text { Anterior superior iliac } \\
\text { spine }\end{array}$} & Lateral condyle & $2 / 3$ & RF, VI & & & & & & RF, VI \\
\hline & Proximal border of & $50 \%$ & RF, VI, VL & & & & & & RF, VI \\
\hline & patella & $60 \%$ & RF, VL & & & & & & \\
\hline & & $2 / 3$ & RF, VL, VI & & & & & & VL \\
\hline & & $3 / 5$ & & $\mathrm{RF}$ & & & & & \\
\hline & & $75 \%$ & & & & $\mathrm{RF}$ & & & \\
\hline & Midpoint of patella & $50 \%$ & RF, VI & & & & & & \\
\hline & $\begin{array}{l}\text { Distal border of } \\
\text { patella }\end{array}$ & $50 \%$ & $\mathrm{RF}$ & & $\mathrm{RF}$ & & & & \\
\hline $\begin{array}{l}\text { Anterior inferior iliac } \\
\text { spine }\end{array}$ & $\begin{array}{l}\text { Proximal border of } \\
\text { patella }\end{array}$ & $50 \%$ & RF, VI & $\mathrm{RF}$ & $\mathrm{RF}$ & & $\mathrm{RF}$ & $\mathrm{RF}$ & RF, VI \\
\hline- & $\begin{array}{l}\text { Proximal border of } \\
\text { patella }\end{array}$ & $\begin{array}{l}10 \mathrm{~cm} \text { proximal to } \\
\text { distal point }\end{array}$ & $\mathrm{RF}$ & & & & & & \\
\hline- & & $\begin{array}{l}15 \mathrm{~cm} \text { proximal to } \\
\text { distal point }\end{array}$ & & $\mathrm{RF}$ & $\mathrm{RF}$ & & & & \\
\hline
\end{tabular}

$M T$ muscle thickness, CSA cross-sectional area, ACSA anatomical cross-sectional area, PCSA physiological cross-sectional area, FL fascicle length, $P A$ pennation angle, $E I$ echo intensity, $R F$ rectus femoris, $V L$ vastus lateralis, $V M$ vastus medialis, $V I$ vastus intermedius 
Measurements of CSA were usually presented in square centimeter. Some studies mentioned to measure the CSA by manually drawing the circumference of the muscle with a cursor $[66,91,127]$. One study normalized the CSA to body mass to represent it as a relative measure of quadriceps muscle [71].

The pennation angle (Ap) was defined as the angle of insertion of muscle fiber fascicles into the deep aponeurosis $[69,97]$. The angle at which fibers in a pennate arrangement are oriented relative to the longitudinal axis varies from muscle to muscle [128]. The pennation angle is proportional to the number of sarcomeres packed in parallel along the aponeurosis and closely related to the force-generating capacity of the muscle [109].

Fascicle length (Lf) was defined as the length of the fascicular path between the insertions of the fascicle into the superficial and deep aponeuroses. In the cases where the fascicle extended outside of the acquired ultrasound image, the length of the missing portion of the fascicle was estimated by extrapolating linearly both the fascicular path, visible in the image, and the aponeurosis $[65,69,97,129$, 130]. Another method of estimating the fascicle length is using a formula: multiplying the muscle thickness times the hypotenuse of the pennation angle inversed [131] or, stated differently, dividing the muscle thickness by the hypotenuse of the pennation angle [99]. These formulae do not account for fascicle curvature [73]. Fascicle length is proportional to the number of sarcomeres arranged in series and the excursion range of the muscle fiber [109].

Echo intensity was defined as the brightness of the image acquired through ultrasound. It is expressed in gray scales (0-255). Some studies used a gray scale analysis for determining the echo intensity [72, 119]. Different programs were used for this analysis, which came down to post-producing the images.

Components and specific measuring points will be given per individual muscle.

\section{Upper leg muscles}

The largest part of information on anatomical landmarks is found on the four bellies of the quadriceps muscle (rectus femoris, vastus lateralis, vastus medialis, vastus intermedius). As common landmarks can be used for the identification of these four muscles, the data gathered are taken together. All measuring points and components of quadriceps muscles are also represented in Table 2 . Besides the quadriceps muscle, the only other upper leg muscle assessed was the biceps femoris (data in the text, not in the table).

At a distance of $60-70 \%$ between the greater trochanter and the popliteal crease, rectus femoris was assessed for muscle thickness [65].
At a distance of $30 \%$ between the greater trochanter and the lateral condyle, vastus medialis was assessed for muscle thickness [84, 131, 132], pennation angle [131] and echo intensity [84, 123]. Vastus lateralis was assessed for muscle thickness [85, 132, 133], fascicle length [85, 133], pennation angle [85] and echo intensity [133]. Rectus femoris was assessed for cross-sectional area [85].

At a distance of $50 \%$ between the greater trochanter and the lateral condyle, rectus femoris was assessed for muscle thickness [41, 75, 84, 89, 95, 131, 134], cross-sectional area $[71,97]$, pennation angle $[89,131]$ and echo intensity [71, 84, 123]. Vastus lateralis was assessed for muscle thickness [70, 80, 84, 88, 89, 99-101, 103, 115, 131, 135], cross-sectional area [88], anatomical cross-sectional area [71, 87, 98, $119,135]$, fascicle length [70, 88, 99-101, 103, 115], pennation angle [70, 88, 89, 99-101, 103, 115] and echo intensity $[71,84,88,119,135,136]$. Vastus intermedius was assessed for muscle thickness [84, 131, 134], pennation angle [131] and echo intensity [84, 136]. Quadriceps (all 4 bellies) were assessed for muscle thickness [137].

At a two-third distance between the greater trochanter and the lateral condyle, rectus femoris was assessed for muscle thickness [67]. Vastus lateralis was assessed for muscle thickness [86], cross-sectional area [86], fascicle length [86], pennation angle [86] and echo intensity [86].

At a distance of $50 \%$ between the greater trochanter and the articular cleft of the knee, rectus femoris and vastus intermedius were assessed for muscle thickness [96].

At a distance of $50 \%$ between the greater trochanter and the proximal border of the patella, rectus femoris was assessed for anatomical cross-sectional area [138] and physiological cross-sectional area [127].

At a two-third distance between the anterior superior iliac spine and the lateral condyle, both rectus femoris and vastus intermedius were assessed for muscle thickness and echo intensity [90].

At a distance of $50 \%$ between the anterior superior iliac spine and the proximal border of the patella, rectus femoris was assessed for muscle thickness [43, 78, 79, 82, 117, 118, $120-122,139,140]$ and echo intensity $[68,83,118,120$, 122]. Vastus intermedius was assessed for muscle thickness [78, 79, 120-122, 139] and echo intensity [120, 122]. Vastus lateralis was assessed for muscle thickness [140].

At a distance of $60 \%$ between the anterior superior iliac spine and the proximal border of the patella, rectus femoris was assessed for muscle thickness [92] as was the vastus lateralis [78].

At a two-third distance between the anterior superior iliac spine and the proximal border of the patella, rectus femoris was assessed for muscle thickness [43, 77, 141]. Vastus lateralis was assessed for muscle thickness [118] and echo intensity [118]. Vastus intermedius was assessed for muscle thickness [77, 141]. 
At a three-fifth distance between the anterior superior iliac spine and the proximal border of the patella, rectus femoris was assessed for cross-sectional area [81].

At a distance of $75 \%$ between the anterior superior iliac spine and the proximal border of the patella, rectus femoris was assessed for physiological cross-sectional area [91].

At a distance of $50 \%$ between the anterior superior iliac spine and the midpoint of the patella, rectus femoris was assessed for muscle thickness [76], as was the vastus intermedius [76].

At a distance of $50 \%$ between the anterior superior iliac spine and the distal border of the patella, rectus femoris was assessed for muscle thickness and anatomical cross-sectional area [93].

At a distance of $50 \%$ between the anterior inferior iliac spine and the proximal border of the patella, rectus femoris was assessed for muscle thickness [88, 94], cross-sectional area [87, 88], anatomical cross-sectional area [119], fascicle length [88], pennation angle [88] and echo intensity [88, 94, 119]. Vastus intermedius was assessed for muscle thickness [94] and echo intensity [94].

At $10 \mathrm{~cm}$ proximal of the proximal border of the patella, rectus femoris was assessed for muscle thickness [142].

At $15 \mathrm{~cm}$ proximal of the proximal border of the patella, rectus femoris was assessed for muscle thickness [74] and anatomical cross-sectional area [143].

At $50 \%$ between the ischial tuberosity and the lateral condyle of the tibia, biceps femoris was assessed for muscle thickness [78].

\section{Lower leg muscles}

The lower leg muscles assessed were the gastrocnemius medialis, the soleus, and the tibialis anterior muscle. One study mentioned that as a bipennate muscle, both the deep and superficial part of the tibialis anterior was measured [97].

At $30 \%$ proximal between the medial condyle of the tibia and the medial malleolus of the fibula, the gastrocnemius medialis was assessed for muscle thickness [78, 103, 115], cross-sectional area [111], anatomical cross-sectional area [108], pennation angle [108, 115], fascicle length [108, 115] and echo intensity [108, 111].

Further measurements use landmarks from the muscle itself.

At $50 \%$ between the proximal and distal tendon insertion of the muscle, gastrocnemius medialis was assessed for muscle thickness [82, 102, 109], fascicle length [69, 97, 102, $110]$ and pennation angle $[69,97,102,107,110]$.

At the most bulky area of the leg, gastrocnemius medialis was assessed for muscle thickness [70, 80, 112, 144], cross-sectional area [145], fascicle length [70, 145] and pennation angle [70, 145].

No studies assessed the gastrocnemius lateralis.

At $30 \%$ proximal between the medial condyle of the tibia and the medial malleolus of the fibula, the soleus was assessed for muscle thickness [78].

At $50 \%$ between the proximal and distal tendon insertion of the muscle, soleus was assessed for pennation angle [146].

At the most bulky area of the leg, soleus was assessed for muscle thickness [112].

For the tibialis anterior, there was only one study, assessing pennation angle and fascicle length at $50 \%$ of muscle length, without giving clear anatomical landmarks [97].

\section{Upper arm muscles}

The upper arm muscles assessed were the biceps brachii and triceps brachii. Less studies used clear anatomical landmarks for the upper arm muscles than for the upper leg muscles.

At $50 \%$ between the acromion and the cubital fossa with the elbows flexed at 90 degrees, biceps brachii was assessed for muscle thickness [113].

At two-thirds between the acromion and the antecubital crease with the arms fully stretched, biceps brachii was assessed for muscle thickness [57].

At 50\% of muscle length (not defined how this is determined), biceps brachii was assessed for fascicle length [97] and pennation angle [97]. This article also mentions that since the fascicles were almost parallel to the superficial aponeurosis, no fascicle length was measured as too much extrapolation was needed [97].

At maximal girth of the upper arm, biceps brachii was assessed for anatomical cross-sectional area [143].

At $40 \%$ between the acromion process of the scapula and the lateral epicondyle of the humerus (starting at the lateral epicondyle), triceps brachii was assessed for muscle thickness [114] and pennation angle [114].

At $40 \%$ between the acromion process of the scapula and the lateral epicondyle of the humerus (starting at the lateral epicondyle), triceps brachii was assessed for muscle thickness [115] and pennation angle [115].

At $50 \%$ between the posterior crista of the acromion and the olecranon triceps brachii was assessed for muscle thickness [116] and pennation angle [116].

\section{Discussion}

It is clear from the multitude of different measuring points that there is little consistency in the current ultrasonographic muscle assessment. To advance US as a routine technique 
Table 3 Consensus proposition, shortcomings in knowledge and protocol listings for patient positioning pre-investigation

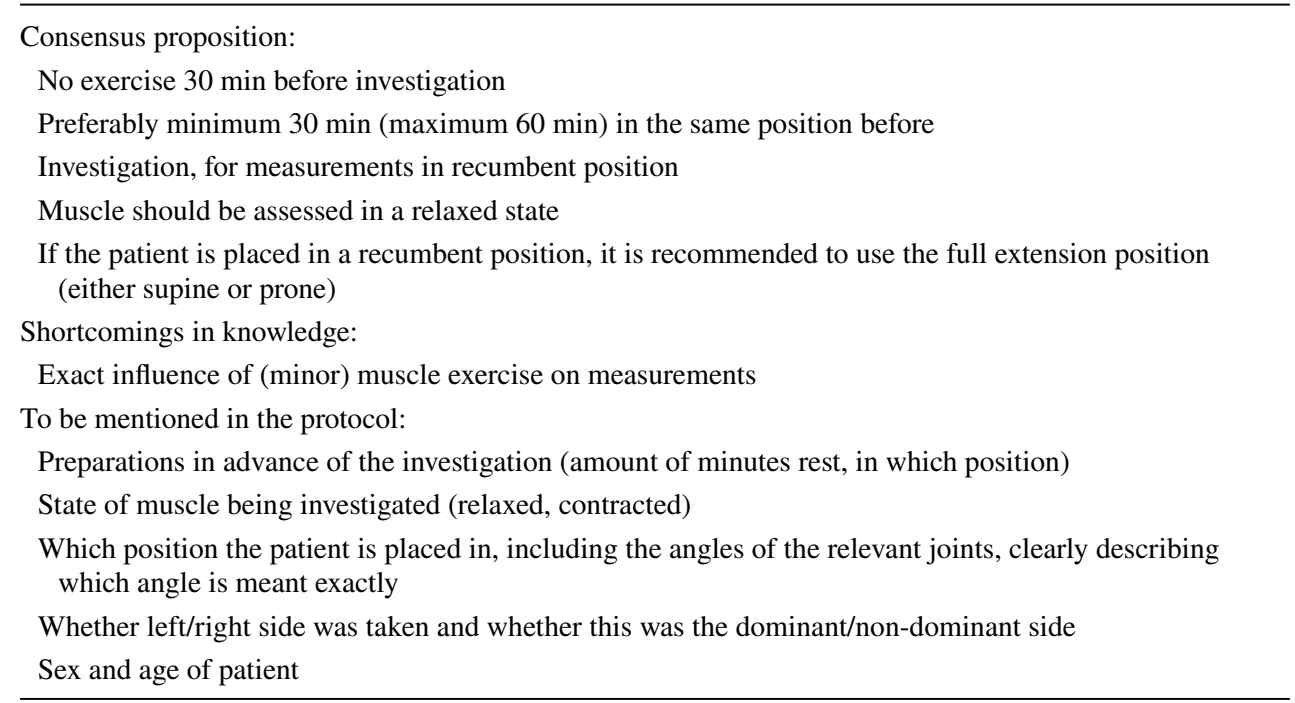

to be used for muscle quality and quantity assessment in old age, standardization is paramount. In the following paragraphs, the possible points of discussion will be addressed regarding the different aspects of measurement. A consensus proposition will be given at the end of each paragraph. Shortcomings in knowledge will also be addressed, as more studies need to be done before able to give recommendations about this subject. We will also propose what information should minimally be mentioned in a study protocol.

\section{Patient positioning pre-investigation}

Since there is a clear difference between relaxed and contracted muscle [147], one state should be chosen. As it is easier to keep a muscle in a relaxed state than in a certain degree of contraction, it seems logical to choose for the former. For the record, it must be said that there is some evidence that contracted muscle correlates better with muscle function than muscle in a relaxed state [147]. Ideally, this should be studied for better intra- and interrater reliability and test-retest reliability. Also the state of contraction before an examination could possibly have an influence on the component measurements. Refraining study subjects from exercises for $24-48 \mathrm{~h}$ is only practical in a purely scientific setting $[74,75]$, not a clinical one, where one would want its patients to exercise as much as possible. Also letting patients performing an amount of maximal contractions [65] could possibly influence measurements. An older study has shown that intensive exercise can give a $15 \%$ increase in water content in a given muscle bulk [148], as also giving way to a possible large measuring error. As it is unclear to what degree minor contractions and light-to-moderate exercises influence muscle volume, it is advised not to let the study subjects do exercises before taking measurements. As there is no clear data on a timeframe, a period of minimal $30 \mathrm{~min}$ is proposed. Ideally, this period is spent laying down.

There are little data on how long patients should be in the same position in order to allow fluid shifts to stabilize [72], or to avoid muscle contraction-induced fluid shifts and muscle blood flow during the measurements [73]. Differences in measurements made in the standing and recumbent positions for example may be due to postural or positional forces acting on muscle shape (for instance joint angle), or due to physiological changes [149]. When going from standing to recumbent position, the most significant changes in thigh muscle size occurred within the first 15-20 min of recumbency, with a stabilization after $60 \mathrm{~min}$ of bed rest $[150,151]$. When measuring a subject in a recumbent position, a minimum of $30 \mathrm{~min}$ and a maximum of $60 \mathrm{~min}$ in the same position prior to the measurements are thus advisable.

The patient positioning in itself is less important for measuring the five components, because depending on what one wants to measure, the positioning could change. However, it must be mentioned that muscle components are significantly different if measured in a standing or in a recumbent position. This is seen more in CSA than in muscle thickness or pennation angle [152]. If the patient is placed in a recumbent position, it is recommended to place the patient in full extension (described above), as in this position, most muscles are relaxed maximally. Mentioning the position in a protocol is thus strongly advised. Regarding dominant/nondominant side, there is some discussion about the relevance of functional asymmetry and strength differences in dominant versus non-dominant side [153]. In non-athletic populations, inter-limb differences in strength are possibly more related to neural factors than pure muscle-related factors [86]. Since this is not clear in literature, it is advised to clearly indicate whether the dominant or non-dominant side is assessed. 
Table 4 Consensus proposition, shortcomings in knowledge and protocol listings for system and system settings

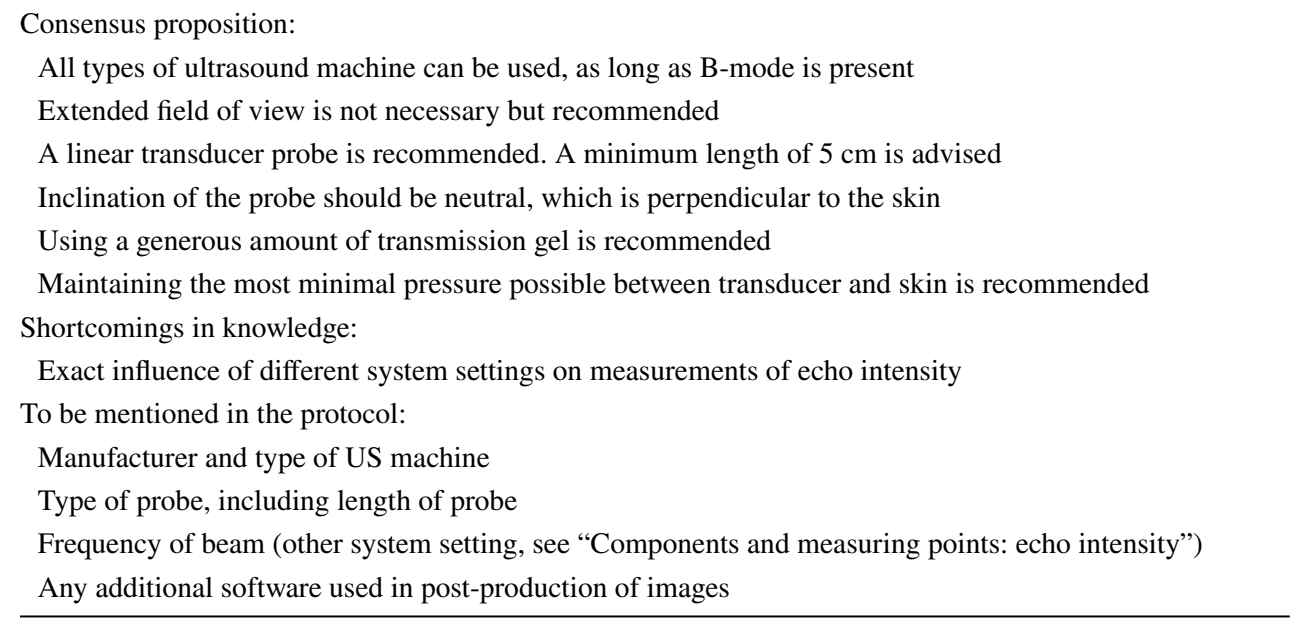

Consensus proposition, shortcomings in knowledge and protocol listings for patient positioning pre-investigation are addressed in Table 3.

\section{System and system settings}

The brand of ultrasonographic machine used is of no relevance for most of the measurements.

Standard B-mode is applied in all studies to visualize the different muscle components and is available on most machines.

Although a curved probe can be used [91], a linear transducer probe is more adapted to assess muscle anatomy.

The length of the transducer is less important for measuring the five main components. However, for assessing crosssectional area and echo intensity, larger (longer) probes can potentially visualize more tissue, which can be helpful if a large muscle bulk is present. Therefore, a minimum length of $5 \mathrm{~cm}$ seems advisable. Extended field of view techniques could help solve this problem.

As no information on inclination of the probe is at hand, it is advised to keep the probe as much perpendicular to the skin as possible.

When applying the probe to the skin, it is important to avoid compression of dermal surface and distortion of muscle surface [72, 104, 154, 155]. Dupont et al. found that applying strong pressure with the ultrasound transducer could flatten the deltoid muscle by $50 \%$ or more and because this error is proportional to muscle size, the absolute error in muscle thickness measurement would be even greater for larger muscles [155]. To minimize both beam loss/scatter and need for dermal/muscle compression, the use of a transmission gel is standard in ultrasonographic investigations. There is no defined standard amount of gel to be used. It is advised to use a generous amount and maintain the minimal pressure possible/necessary between transducer and the skin [155].

The frequency of the transducer beam is less relevant for measuring components, except for assessing echo intensity. However, the higher the frequency, the better is the visualization of anatomical structures. Using ultrasound, there is therefore a constant compromise between image resolution and depth of penetration of the sound waves. Higher frequency transducers provide better spatial resolution, but these transducers have a shallower depth of penetrance than a lower frequency transducer [156].

To our knowledge, other system settings do not seem to be relevant for measuring the components, except for assessing echo intensity. Depth focus, general gain, time-gain compensation, dynamic range, etc. can be set in order to have the best possible view of the muscle that is to be assessed.

The use of software for post-processing and measurement is less relevant for measuring the components, except for assessing echo intensity. Most modern machines have software that allows measurements to be directly done during the investigation; otherwise, all other picture post-processing software can be used.

Measuring echo intensity is dependent on many factors. These are discussed under the paragraphs "Components" and "Measuring points".

Consensus proposition, shortcomings in knowledge and protocol listings for system and system settings are addressed in Table 4.

\section{Components}

The five components that can be easily measured when assessing muscle components are already mentioned: muscle thickness, pennation angle, fascicle length, echo intensity and cross-sectional area. The technique to measure these 
items is relative easy. The most difficult part is to define where to do the measurements. As there is no information available on the most ideal location within the muscle, there exists a multitude of measuring points. Since no scientific substantiated "best" point can be defined, consensus locations will be provided for each muscle described in literature.

It seems logical to use the thickest zone of a muscle when wanting to assess muscle thickness, as this will be the place that the muscle will generate the most contractive power. However, there are no studies to our knowledge that have looked at the evolution of muscle thickness throughout a specific muscle. Therefore, we advise different approaches for different kinds of muscles.
Theoretically, muscles like the four bellies from the quadriceps are the thickest at $50 \%$ of their length, measured from tendon to tendon [157]. Therefore, we propose to mark and use the point at $50 \%$ of the muscle's length (50\% rule).

Unfortunately, not every muscle is shaped like the bellies of the quadriceps, e.g., the gastrocnemius medialis. This poses a certain difficulty, and a possible reason for measurement bias. In the literature, in this type of muscle the "maximal bulk" was most often noted as measuring point. We propose that in more asymmetrical muscles, the point of 50\% length between tendons is visualized, and then 4 additional points are checked: at 30, 40, 60 and $70 \%$ length between

Table 5 Consensus proposition, shortcomings in knowledge and protocol listings for components

\section{Consensus proposition:}

Five components can be measured: muscle thickness, pennation angle, fascicle length, echo intensity and cross-sectional area

Measurements are ideally done at maximal muscle bulk

Depending on muscle anatomy, different techniques are advised for determining maximal muscle bulk

Panoramic vision and extended-field-of-view software are not absolutely necessary but recommended

In pennate muscles, measuring physiological CSA rather than anatomical CSA is recommended

When the fascicle length cannot be directly measured, it can be calculated using the standard formula

When measuring echo intensity, all system settings need to be kept the same. Currently, no proposition for specific system settings based upon literature can be done for echo intensity

Shortcomings in knowledge:

Exact point of maximal muscle thickness for each muscle

Changes of the main components (MT, CSA, FL, PA, EI) throughout the muscle bulk

A good measure for comparing echo intensity between different US machines/systems

To be mentioned in the protocol:

The muscle that is assessed, with inclusion of the anatomical landmarks that are used and the exact point in between the landmarks. If not the midpoint, clearly describe whether the proximal or distal end is meant

The components that are measured. If CSA is measured, define if anatomical or physiological CSA is meant

Total length of muscle (to calculate relative muscle thickness values)

The technique that is used to determine the position of maximal bulk

Table 6 Proposed anatomical landmarks for each muscle discussed

\begin{tabular}{|c|c|c|c|}
\hline & Proximal landmark & Distal landmark & Asymmetry \\
\hline \multicolumn{4}{|l|}{ Lower limb } \\
\hline Rectus femoris & Greater trochanter & Proximal border of patella & Minimal \\
\hline Vastus lateralis & Greater trochanter & Proximal border of patella & Minimal \\
\hline Vastus medialis & Greater trochanter & Proximal border of patella & Minimal \\
\hline Vastus intermedius & Greater trochanter & Proximal border of patella & Minimal \\
\hline Biceps femoris (long head) & Ischial tuberosity & Proximal head of fibula & Minimal \\
\hline Tibialis anterior & Lateral condyle (anterior) of tibia & US-measurement dependant & Minimal \\
\hline Gastrocnemius (medialis) & Medial condyle (posterior) of the femur & US-measurement dependant & Minimal \\
\hline Gastrocnemius (lateralis) & Medial condyle (posterior) of the femur & US-measurement dependant & Minimal \\
\hline Soleus & Proximal head of fibula (posterior part) & Posterior superior part of calcaneus & Yes \\
\hline \multicolumn{4}{|l|}{ Upper limb } \\
\hline Biceps brachii & $\begin{array}{l}\text { Anterior part of acromion process (acromio- } \\
\text { clavicular joint) }\end{array}$ & Elbow crease where tendon can be palpated & Yes \\
\hline Triceps brachii & Most lateral distal part of acromion & Tip of olecranon & Yes \\
\hline
\end{tabular}


tendons. Then, the maximal muscle thickness can be chosen from these measurements. Without having large studies that provide reference data per muscle, this will have to be done per muscle, per patient. In muscles with severe asymmetry, e.g., biceps femoris, it is best to specify which part of the muscle is assessed, e.g., biceps femoris-long head.

Another variety is the muscle with a long tendon, which has no clear anatomical landmarks to indicate where the muscle bulk ends, e.g., tibialis anterior. In these muscles, it is advised to locate the proximal and distal border of the muscle through ultrasound before referring to the $50 \%$ rule.

Noting down the $100 \%$ length of the muscle from tendon to tendon is advised, as a longer muscle can potentially generate more power. It is not known whether absolute muscle thickness or relative muscle thickness (=muscle thickness/ length of the muscle) is more representative [158]. To be complete, there could be other reasons to choose for a different measurement site in specific muscles. Ticinesi et al. [86] suggested to use the distal point of $65 \%$ of the length of the vastus lateralis, as this site is most free of vessels and muscle biopsies can be easily made, avoiding major vessels and nerves. However, without specific arguments for clinical correlates, this is not recommended. For this, studies are needed.

For measuring the maximal thickness of a muscle, it is advised not only to take the midpoint of the muscle in between the tendons, but at this point of the longitudinal axis, to use the point at $50 \%$ between the medial and lateral border of the muscle bulk. This will also have to be visualized through ultrasound and marked for easy Ref. [86].

The argument of using the thickest zone of the muscle bulk is also valid for measuring the cross-sectional area. One of the disadvantages of this point could be that in some cases it will be difficult to get a complete image of the CSA, in the case of a large muscle bulk. Panoramic vision and extendedfield-of-view software that are almost standard on most US machines can nullify this problem. Also, since it is unknown how the muscle volume diminishes exactly towards the tendons, submaximal measurements do not weigh up to the maximal CSA. For this, studies are needed.

As said, cross-sectional area can be divided into anatomical CSA and physiological CSA. The former underestimates the number of total fibers in a pennated muscle, so in these types of muscles the use of the PCSA is advised. In nonpennate muscles, the ASCA is the same as the PCSA.

As there is no hard information available to our knowledge about the pattern of echo intensity or pennation angle throughout a given muscle-homogenous versus heterogenous [159] - the proposition is to take all measurements (including the fascicle length) at the point of maximal bulk, as discussed for muscle thickness and CSA.

There is no doubt that echo intensity is an important parameter. As a parameter of fatty infiltration of the muscle (myosteatosis), it helps unravel an important aspect of the process of sarcopenia. Myosteatosis is linked with increased mortality in specific populations [20,160-162], with a strong need for further clinical investigation. However, the standardisation and comparability of measurements of echo intensity between different US system brands are appalling. Defined as the brightness of the image and expressed in gray scales, there are many factors (system relates) that influence the image. A small difference in beam frequency or gain —or many other settings — can give completely different results, and comparing system settings between different manufacturers is currently impossible. No good calibration model exists to date. Even the age of a probe can influence results by influencing the strength of the beam emitted. Also, although the analysis of muscle tissue acquired via biopsy suggests that echogenicity is more strongly associated with intramuscular adipose tissue rather than fibrosis [163, 164], biopsies are still needed to determine the percentage both components [165]. More studies are needed on both the comparability between systems and the differentiation of fat and fibrosis. Ideally, a universally accepted calibration dummy will be developed in the near future.

Consensus proposition, shortcomings in knowledge and protocol listings for components are addressed in Table 5.

\section{Measuring points}

For the appendicular muscle described, we refer to Table 6 for an overview of the proposed anatomical landmarks. These landmarks were selected in view of the discussion above, and with regard to the possible use of good-identifiable anatomical landmarks. As discussed in the paragraph about muscle thickness, some muscles show an asymmetry, either in length, width, or both. Table 6 also indicates whether a certain asymmetry exists and, thus, caution has to be taken in use of the $50 \%$ rule. When there are multiple origins, or if there is a larger area of insertion, the most identifiable/representative landmark is chosen. Future studies will have to confirm the usability of these landmarks.

\section{Method of measurement}

As a short overview, the proposed procedure of measurement will be shortly addressed. This procedure is based on the discussion above. It will be represented schematically as a sort of checklist.

- Place the patient in the desired position, preferably 30 min before investigation.

- Select the muscle that is to be assessed, and check for the desired technique to locate the maximal muscle bulk. 
- Use the appropriate technique and the anatomical landmarks provided to locate the longitudinal measuring point. Mark this with a dermographic pencil.

- Locate the medial and lateral side of the muscle and mark these with a dermographic pencil. Use the middle point of these marks. Now the correct measuring point is found.

- Keeping the transducer probe in a longitudinal direction in line with the muscle fiber fascicles. At this position, measure muscle thickness (from aponeurosis to aponeurosis), pennation angle (angle of muscle fiber fascicles into the deep aponeurosis) and fascicle length.

- Turn the transducer probe $90^{\circ}$. At this position, measure cross-sectional area. As this will probably not be a perfect ellipse, the circumference of the muscle can be manually drawn with a cursor. Use this maximal area to also measure echo intensity.

- Repeat all measurements three times and use the mean value of these measurements.

\section{Limitations}

There are some limitations to this review. Although the search strategy was very broad, we have focused on appendicular muscle mass, and have not included facial, thoracic, abdominal and pelvic muscle. Also the incidental reports from smaller muscle groups from the hand were not included, because too little information was available per muscle. The muscle groups that were not included in this review could be the subject of future investigations.

Also some ultrasound-based techniques were not included in this review, such as elastography. This could also be the subject of a review in itself.

\section{Future direction}

The ultimate goal would be to have not only a standardized way to assess muscle characteristics, but also to have a workable algorithm to diagnose sarcopenia using ultrasound. For this, some barriers have to be taken.

First of all more insights should be gained into the agerelated evolution of the different muscle characteristics. It seems reasonable to assume that muscle thickness and cross-sectional area for instance will decrease with age, but perhaps the pennation angle will start shifting before any thickness or volume dimensions change. This is important because making a diagnosis as early as possible also means that treatment can start earlier.

Secondly, reference values of a 'normal population' are lacking. In 2016, Minetto et al. [82] have already done some work on this, using muscle thickness values 2 standard deviations below the gender-specific means of a sample of younger subjects to diagnose sarcopenia. In this regard, one could discuss about using one set of cutoff points derived from a younger population, or if age-related reference values should be used. It is also clear that all reference points should be specific for each muscle. This means that every muscle should be examined separately, because cutoff points for, e.g., the vastus lateralis are different than those for the gastrocnemius.

Thirdly, it is too early to say if diagnosing sarcopenia with ultrasound should be done by a composite score or by a single measurement. In this stage, it seems advisable to measure all muscle characteristics and link this with diagnostic criteria that are currently used (e.g., BIA, DEXA). In a later stage, perhaps it will become clear that certain ultrasoundbased characteristics are more important than others. For this, more studies have to be done.

Fourthly, the measurements proposed in this manuscript are not exhaustive. Perhaps other parameters are equal or more important, e.g., elastography of the muscle or tendon. Although an area that should certainly be explored, too little is known of this in the context of sarcopenia to currently make any statements.

In summary, there is certainly a dynamic towards using ultrasound for diagnosing sarcopenia, but more studies need to be done, preferentially starting with the creation of reference values.

\section{Conclusion}

To compare studies and advance the use of ultrasound in the assessment of sarcopenia, certain standardization has to be done. However, it is not yet clear which measuring points are more relevant than others. Therefore, the recommendations proposed in this review should not be regarded as set in stone. They are rather intended as a reference point or as a guideline, from which comparative studies can be initiated. Future directions can certainly involve changing some of the recommendations, if there is new evidence to support these changes. The limitations of this review are clear since there are a lot of unknown factors: the exact spreading pattern and evolution of the different architectural components throughout the muscle, which muscles or muscle measurements are most clinically relevant, influence of position of the patient, influence of pre-investigation activity, etcetera. Therefore, this consensus approach is used as a starting point. Hopefully, this way future studies will have an extra support to build upon. Also, this way interested researchers can collaborate towards an ultrasonographic diagnosis of sarcopenia.

In conclusion, this review offers a guideline for investigators wanting to set up a study using ultrasound in muscle assessment. Studies in clinical settings are needed to validate the effectiveness of these propositions. 


\section{Compliance with ethical standards}

Conflict of interest The authors declare that they have no conflict of interest.

Ethical approval This article does not contain any studies with human participants performed by any of the authors.

\section{References}

1. Beaudart C et al (2014) Sarcopenia: burden and challenges for public health. Arch Public Health 72(1):45

2. Beaudart $\mathrm{C}$ et al (2017) Health outcomes of sarcopenia: a systematic review and meta-analysis. PLoS ONE 12(1):e0169548

3. Cruz-Jentoft AJ et al (2010) Sarcopenia: European consensus on definition and diagnosis: report of the European Working Group on sarcopenia in older people. Age Ageing 39(4):412-423

4. Muscaritoli M et al (2010) Consensus definition of sarcopenia, cachexia and pre-cachexia: joint document elaborated by Special Interest Groups (SIG) "cachexia-anorexia in chronic wasting diseases" and "nutrition in geriatrics". Clin Nutr 29(2):154-159

5. Fielding RA et al (2011) Sarcopenia: an undiagnosed condition in older adults. Current consensus definition: prevalence, etiology, and consequences. International working group on sarcopenia. J Am Med Dir Assoc 12(4):249-256

6. Morley JE et al (2011) Sarcopenia with limited mobility: an international consensus. J Am Med Dir Assoc 12(6):403-409

7. Dam TT et al (2014) An evidence-based comparison of operational criteria for the presence of sarcopenia. J Gerontol A Biol Sci Med Sci 69(5):584-590

8. Chen LK et al (2014) Sarcopenia in Asia: consensus report of the Asian Working Group for Sarcopenia. J Am Med Dir Assoc 15(2):95-101

9. Beaudart $C$ et al (2015) Estimation of sarcopenia prevalence using various assessment tools. Exp Gerontol 61:31-37

10. Baumgartner RN et al (1998) Epidemiology of sarcopenia among the elderly in New Mexico. Am J Epidemiol 147(8):755-763

11. Delmonico MJ et al (2007) Alternative definitions of sarcopenia, lower extremity performance, and functional impairment with aging in older men and women. J Am Geriatr Soc 55(5):769-774

12. Newman AB et al (2003) Sarcopenia: alternative definitions and associations with lower extremity function. J Am Geriatr Soc 51(11):1602-1609

13. Chien MY, Huang TY, Wu YT (2008) Prevalence of sarcopenia estimated using a bioelectrical impedance analysis prediction equation in community-dwelling elderly people in Taiwan. J Am Geriatr Soc 56(9):1710-1715

14. Janssen I et al (2004) Skeletal muscle cutpoints associated with elevated physical disability risk in older men and women. Am J Epidemiol 159(4):413-421

15. Janssen I, Heymsfield SB, Ross R (2002) Low relative skeletal muscle mass (sarcopenia) in older persons is associated with functional impairment and physical disability. J Am Geriatr Soc 50(5):889-896

16. Barbat-Artigas $S$ et al (2012) How to assess functional status: a new muscle quality index. J Nutr Health Aging 16(1):67-77

17. Newman AB et al (2003) Strength and muscle quality in a wellfunctioning cohort of older adults: the Health, Aging and Body Composition Study. J Am Geriatr Soc 51(3):323-330

18. Goodpaster BH et al (2006) The loss of skeletal muscle strength, mass, and quality in older adults: the health, aging and body composition study. J Gerontol A Biol Sci Med Sci 61(10):1059-1064
19. Perkisas $\mathrm{S}$ et al (2016) Physiological and architectural changes in the ageing muscle and their relation to strength and function in sarcopenia. Eur Geriatr Med 7(3):201-206. https://doi. org/10.1016/j.eurger.2015.12.016

20. Perkisas S, De Cock AM, Verhoeven V, Vandewoude M (2017) Intramuscular adipose tissue and the functional components of sarcopenia in hospitalized geriatric patients. Geriatrics 2(1):11. https://doi.org/10.3390/geriatrics2010011

21. Reinders I et al (2016) Muscle quality and myosteatosis: novel associations with mortality risk: the Age, Gene/Environment Susceptibility (AGES)-Reykjavik Study. Am J Epidemiol 183(1):53-60

22. Buckinx F et al (2018) Pitfalls in the measurement of muscle mass: a need for a reference standard. J Cachexia Sarcopenia Muscle 9(2):269-278

23. Yamada $Y$ et al (2017) Electrical properties assessed by bioelectrical impedance spectroscopy as biomarkers of age-related loss of skeletal muscle quantity and quality. J Gerontol A Biol Sci Med Sci 72(9):1180-1186

24. Bartels EM, Sorensen ER, Harrison AP (2015) Multi-frequency bioimpedance in human muscle assessment. Physiol Rep 3(4):e12354

25. Beaudart $C$ et al (2016) Sarcopenia in daily practice: assessment and management. BMC Geriatr 16(1):170

26. Goodpaster BH et al (2000) Skeletal muscle attenuation determined by computed tomography is associated with skeletal muscle lipid content. J Appl Physiol (1985) 89(1):104-110

27. Heymsfield SB et al (2014) Assessing skeletal muscle mass: historical overview and state of the art. J Cachexia Sarcopenia Muscle 5(1):9-18

28. Fischer MA et al (2014) Dixon-based MRI for assessment of muscle-fat content in phantoms, healthy volunteers and patients with achillodynia: comparison to visual assessment of calf muscle quality. Eur Radiol 24(6):1366-1375

29. Pfirrmann CW et al (2004) Assessment of fat content in supraspinatus muscle with proton MR spectroscopy in asymptomatic volunteers and patients with supraspinatus tendon lesions. Radiology 232(3):709-715

30. Prompers JJ et al (2006) Dynamic MRS and MRI of skeletal muscle function and biomechanics. NMR Biomed 19(7):927-953

31. Valkovic L, Chmelik M, Krssak M (2017) In-vivo(31)P-MRS of skeletal muscle and liver: a way for non-invasive assessment of their metabolism. Anal Biochem 529:193-215

32. Mourtzakis M et al (2008) A practical and precise approach to quantification of body composition in cancer patients using computed tomography images acquired during routine care. Appl Physiol Nutr Metab 33(5):997-1006

33. Shen $\mathrm{W}$ et al (2004) Total body skeletal muscle and adipose tissue volumes: estimation from a single abdominal cross-sectional image. J Appl Physiol (1985) 97(6):2333-2338

34. Janssen I et al (2000) Skeletal muscle mass and distribution in 468 men and women aged 18-88 yr. J Appl Physiol (1985) 89(1):81-88

35. Pillen S, van Alfen N (2011) Skeletal muscle ultrasound. Neurol Res 33(10):1016-1024

36. Passmore E, Lai A, Sangeux M et al (2017) Application of ultrasound imaging to subject-specific modelling of the human musculoskeletal system. Meccanica 52:665. https:// doi.org/10.1007/s11012-016-0478-z

37. Abe T et al (2016) Ultrasound-derived forearm muscle thickness is a powerful predictor for estimating DXA-derived appendicular lean mass in Japanese older adults. Ultrasound Med Biol 42(9):2341-2344

38. Takai Y et al (2014) Applicability of ultrasound muscle thickness measurements for predicting fat-free mass in elderly population. J Nutr Health Aging 18(6):579-585 
39. Takai Y et al (2013) Validity of ultrasound muscle thickness measurements for predicting leg skeletal muscle mass in healthy Japanese middle-aged and older individuals. J Physiol Anthropol 32:12

40. Abe $\mathrm{T}$ et al (2015) Validity of ultrasound prediction equations for total and regional muscularity in middle-aged and older men and women. Ultrasound Med Biol 41(2):557-564

41. Thomaes T et al (2012) Reliability and validity of the ultrasound technique to measure the rectus femoris muscle diameter in older CAD-patients. BMC Med Imaging 12:7

42. Sanada $K$ et al (2006) Prediction and validation of total and regional skeletal muscle mass by ultrasound in Japanese adults. Eur J Appl Physiol 96(1):24-31

43. Tandon $\mathrm{P}$ et al (2016) A model to identify sarcopenia in patients with cirrhosis. Clin Gastroenterol Hepatol 14(10):1473-1480 e3

44. Reeves ND, Maganaris CN, Narici MV (2004) Ultrasonographic assessment of human skeletal muscle size. Eur J Appl Physiol 91:116-118

45. Nijholt $\mathrm{W}$ et al (2017) The reliability and validity of ultrasound to quantify muscles in older adults: a systematic review. J Cachexia Sarcopenia Muscle 8(5):702-712

46. Mourtzakis M, Wischmeyer P (2014) Bedside ultrasound measurement of skeletal muscle. Curr Opin Clin Nutr Metab Care 17(5):389-395

47. Haberfehlner $\mathrm{H}$ et al (2016) Freehand three-dimensional ultrasound to assess semitendinosus muscle morphology. J Anat 229:591-599

48. Martin DC et al (2001) Comparing human skeletal muscle architectural parameters of cadavers with in vivo ultrasonographic measurements. J Anat 199:429-434

49. Tosovic D et al (2016) Anatomy of the long head of biceps femoris: an ultrasound study. Clin Anat 29(6):738-745

50. Kellis E et al (2009) Validity of architectural properties of the hamstring muscles: correlation of ultrasound findings with cadaveric dissection. J Biomech 42(15):2549-2554

51. Infantolino BW, Challis JH (2016) Evaluation of a simple method for determining muscle volume in vivo. J Biomech 49(9):1973-1975

52. Tillquist $\mathrm{M}$ et al (2014) Bedside ultrasound is a practical and reliable measurement tool for assessing quadriceps muscle layer thickness. J Parenter Enter Nutr 38(7):886-890

53. English C, Fisher L, Thoirs K (2012) Reliability of real-time ultrasound for measuring skeletal muscle size in human limbs in vivo: a systematic review. Clin Rehabil 26(10):934-944

54. Miyatani M et al (2004) The accuracy of volume estimates using ultrasound muscle thickness measurements in different muscle groups. Eur J Appl Physiol 91(2):264-272

55. Nijholt W et al (2018) Response to: "The use of ultrasound for the estimation of muscle mass: one site fits most?". J Cachexia Sarcopenia Muscle 9:627-628

56. Reimers CD, Harder T, Saxe H (1998) Age-related muscle atrophy does not affect all muscles and can partly be compensated by physical activity: an ultrasound study. J Neurol Sci 159(1):60-66

57. Arts IMP et al (2007) Rise and fall of skeletal muscle size over the entire life span. J Am Geriatr Soc 55:1150-1152

58. Arts IMP et al (2010) Normal values for quantitative muscle ultrasonography in adults. Muscle Nerve 41(1):32-41

59. Verhulst FV et al (2011) Quantitative ultrasound of lower leg and foot muscles: feasibility and reference values. Foot Ankle Surg Off J Eur Soc Foot Ankle Surg 17(3):145-149

60. Maurits NM et al (2002) Quantitative muscle ultrasound analysis in healthy adults: weight- and age-related normal values. Neuromuscular Disord 12(7):776
61. Ticinesi A et al (2017) Muscle ultrasound and sarcopenia in older individuals: a clinical perspective. J Am Med Dir Assoc 18(4):290-300

62. Welch $\mathrm{C}$ et al (2018) Acute sarcopenia secondary to hospitalisation-an emerging condition affecting older adults. Aging Dis $9(1): 151-164$

63. van de Glind EM et al (2012) Search filters to identify geriatric medicine in Medline. J Am Med Inform Assoc 19(3):468-472

64. Ouzzani M et al (2016) Rayyan-a web and mobile app for systematic reviews. Syst Rev 5(1):210

65. Ema $R$ et al (2013) In vivo measurement of human rectus femoris architecture by ultrasonography: validity and applicability. Clin Physiol Funct Imaging 33:267-273

66. de Bruin PF et al (1997) Size and strength of the respiratory and quadriceps muscles in patients with chronic asthma. Eur Respir J 10:59-64

67. Correa CS et al (2016) Effects of strength training, detraining and retraining in muscle strength, hypertrophy and functional tasks in older female adults. Clin Physiol Funct Imaging 36(4):306-310

68. Harris-Love MO et al (2016) Ultrasound estimates of muscle quality in older adults: reliability and comparison of Photoshop and ImageJ for the grayscale analysis of muscle echogenicity. PeerJ 4:e1721

69. Narici MV et al (2003) Effect of aging on human muscle architecture. J Appl Physiol (Bethesda, Md.: 1985) 95(6):2229-2234

70. Kaya A et al (2013) Ultrasonographic evaluation of the muscle architecture in patients with systemic lupus erythematosus. Clin Rheumatol 32(8):1155-1160

71. Kleinberg CR et al (2016) Influence of lower extremity muscle size and quality on stair-climb performance in career firefighters. J Strength Cond Res 30(6):1613-1618

72. da Silva RP et al (2017) Effect of strength training on sleep apnea severity in the elderly: study protocol for a randomized controlled trial. Trials 18(1):489

73. Akima $\mathrm{H}$ et al (2017) Relationship between quadriceps echo intensity and functional and morphological characteristics in older men and women. Arch Gerontol Geriatr 70:105-111

74. Bemben DA, Langdon DB (2002) Relationship between estrogen use and musculoskeletal function in postmenopausal women. Maturitas 42:119-127

75. Baroni BM et al (2013) Time course of neuromuscular adaptations to knee extensor eccentric training. Int J Sports Med 34(10):904-911

76. Gerovasili V et al (2009) Electrical muscle stimulation preserves the muscle mass of critically ill patients: a randomized study. Crit Care 13:R161

77. Guerreiro AC et al (2017) Bedside ultrasound of quadriceps to predict rehospitalization and functional decline in hospitalized elders. Front Med 4:122

78. Ikezoe T et al (2011) Age-related muscle atrophy in the lower extremities and daily physical activity in elderly women. Arch Gerontol Geriatr 53(2):e153-e157

79. Ikezoe T et al (2012) Associations of muscle stiffness and thickness with muscle strength and muscle power in elderly women. Geriatr Gerontol Int 12:86-92

80. Kara $\mathrm{M}$ et al (2015) Does vitamin D affect muscle strength and architecture? An isokinetic and ultrasonographic study. Asia Pac J Clin Nutr 26(1):85-88

81. Seymour JM et al (2009) Ultrasound measurement of rectus femoris cross-sectional area and the relationship with quadriceps strength in COPD. Thorax 64:418-423

82. Minetto MA et al (2016) Ultrasound-based detection of low muscle mass for diagnosis of sarcopenia in older adults. PM\&R J Inj Funct Rehabil 8(5):453-462 
83. Mota JA, Stock MS (2017) Rectus femoris echo intensity correlates with muscle strength, but not endurance, in younger and older men. Ultrasound Med Biol 43(8):1651-1657

84. Rech A et al (2014) Echo intensity is negatively associated with functional capacity in older women. Age 36:9708

85. Rieder F et al (2015) Alpine Skiing With total knee ArthroPlasty (ASWAP): muscular adaptations. Scand J Med Sci Sports 25:26-32

86. Ticinesi A, Narici MV, Lauretani F et al (2018) Assessing sarcopenia with vastus lateralis muscle ultrasound: an operative protocol. Aging Clin Exp Res. https://doi.org/10.1007/s4052 0-018-0958-1

87. Scanlon, T.C., et al., Muscle architecture and strength: adaptations to short-term resistance training in older adults. Vol. 49. 2014. 584-92

88. Mangine GT et al (2014) Bilateral differences in muscle architecture and increased rate of injury in national basketball association players. J Athl Train 49(6):794-799

89. Marin PJ et al (2013) Effects of whole-body vibration on muscle architecture, muscle strength, and balance in stroke patients a randomized controlled trial. Am J Phys Med Rehabil 92(10):881-888

90. Cruz-Montecinos C et al (2016) Sonographic measurement of the quadriceps muscle in patients with chronic obstructive pulmonary disease: functional and clinical implications. J Ultrasound Med 35(11):2405-2412

91. Hammond $\mathrm{K}$ et al (2014) Validity and reliability of rectus femoris ultrasound measurements: comparison of curved-array and linear-array transducers. J Rehabil Res Dev 51:1155-1164

92. Mueller $\mathrm{N}$ et al (2016) Can sarcopenia quantified by ultrasound of the rectus femoris muscle predict adverse outcome of surgical intensive care unit patients as well as frailty? A prospective, observational cohort study. Ann Surg 264:1116-1124

93. Berger J et al (2015) Rectus femoris (RF) ultrasound for the assessment of muscle mass in older people. Arch Gerontol Geriatr 61:33-38

94. Fukumoto Y et al (2012) Skeletal muscle quality assessed from echo intensity is associated with muscle strength of middle-aged and elderly persons. Eur J Appl Physiol 112(4):1519-1525

95. Shimizu $\mathrm{S}$ et al (2006) The relationship between cardiac muscle, skeletal muscle mass, and vessel structure in elderly women. Jpn J Phys Fit Sports Med 55:213-216

96. Nakatani $M$ et al (2016) Validity of muscle thickness-based prediction equation for quadriceps femoris volume in middle-aged and older men and women. Eur J Appl Physiol 116(11):2125-2133

97. de Boer MD et al (2008) Effect of 5 weeks horizontal bed rest on human muscle thickness and architecture of weight bearing and non-weight bearing muscles. Eur J Appl Physiol 104(2):401-407

98. Lixandrão ME et al (2016) Time course of resistance traininginduced muscle hypertrophy in the elderly. J Strength Cond Res 30:159-163

99. Alegre LM et al (2006) Effects of dynamic resistance training on fascicle length and isometric strength. J Sports Sci 24(5):501-508

100. Malas FÜ et al (2013) Effects of different strength training on muscle architecture: clinical and ultrasonographic evaluation in knee osteoarthritis. PM\&R 5:655-662

101. de Oliveira Melo M et al (2015) Effects of neuromuscular electrical stimulation and low-level laser therapy on the muscle architecture and functional capacity in elderly patients with knee osteoarthritis: a randomized controlled trial. Clin Rehabil 29:570-580
102. Karamanidis K, Arampatzis A (2005) Mechanical and morphological properties of different muscle-tendon units in the lower extremity and running mechanics: effect of aging and physical activity. J Exp Biol 208:3907-3923

103. Fukutani A, Kurihara T (2015) Comparison of the muscle fascicle length between resistance-trained and untrained individuals: cross-sectional observation. Springerplus 4:341

104. Engelina S et al (2014) Ultrasound investigation of vastus medialis oblique muscle architecture: an in vivo study. Clin Anat (New York, NY) 27(7):1076-1084

105. Akazawa $\mathrm{N}$ et al (2017) Relationships between intramuscular fat, muscle strength and gait independence in older women: a cross-sectional study. Geriatr Gerontol Int 17(10):1683-1688

106. Selva Raj I, Bird SR, Shield AJ (2017) Ultrasound measurements of skeletal muscle architecture are associated with strength and functional capacity in older adults. Ultrasound Med Biol 43:586-594

107. Erskine RM et al (2017) The individual and combined effects of obesity- and ageing-induced systemic inflammation on human skeletal muscle properties. Int J Obes (2005) 41(1):102-111

108. Gerstner GR et al (2017) Neural and muscular contributions to the age-related reductions in rapid strength. Med Sci Sports Exerc 49(7): 1331-1339

109. Atkinson RA et al (2010) Effects of testosterone on skeletal muscle architecture in intermediate-frail and frail elderly men. J Gerontol Ser A Biomed Sci Med Sci 65:1215-1219

110. Morse CI et al (2005) In vivo physiological cross-sectional area and specific force are reduced in the gastrocnemius of elderly men. J Appl Physiol (1985) 99(3):1050-1055

111. Rosenberg JG et al (2014) Reliability of panoramic ultrasound imaging to simultaneously examine muscle size and quality of the medial gastrocnemius. Muscle Nerve 49(5):736-740

112. Fujiwara $\mathrm{K}$ et al (2010) Regular heel-raise training focused on the soleus for the elderly: evaluation of muscle thickness by ultrasound. J Physiol Anthropol 29(1):23-28

113. Ohata $\mathrm{K}$ et al (2006) Measurement of muscle thickness as quantitative muscle evaluation for adults with severe cerebral palsy. Phys Ther 86(9):1231-1239

114. Kawakami Y, Abe T, Fukunaga T (1993) Muscle-fiber pennation angles are greater in hypertrophied than in normal muscles. J Appl Physiol 74:2740-2744

115. Kubo K et al (2003) Muscle architectural characteristics in women aged 20-79 years. Med Sci Sports Exerc 35:39-44

116. Ojanen T, Rauhala T, Häkkinen K (2007) Strength and power profiles of the lower and upper extremities in master throwers at different ages. J Strength Cond Res 21(1):216-222

117. Stock MS et al (2016) Evidence of muscular adaptations within four weeks of barbell training in women. Human Mov Sci $45: 7-22$

118. Nijboer-Oosterveld J, Van Alfen N, Pillen S (2011) New normal values for quantitative muscle ultrasound: obesity increases muscle echo intensity. Muscle Nerve 43:142-143

119. Fragala MS et al (2014) Biomarkers of muscle quality: N-terminal propeptide of type III procollagen and C-terminal agrin fragment responses to resistance exercise training in older adults. J Cachexia Sarcopenia Muscle 5(2):139-148

120. Taniguchi $M$ et al (2017) Increase in echo intensity and extracellular-to-intracellular water ratio is independently associated with muscle weakness in elderly women. Eur J Appl Physiol 117(10):2001-2007

121. Yamada M et al (2017) Differential characteristics of skeletal muscle in community-dwelling older adults. J Am Med Dir Assoc 18(9):807.e9-807.e16

122. Ye X, Wang MJ, Xiao H (2017) Echo intensity of the rectus femoris in stable COPD patients. Int J Chronic Obstr Pulm Dis 12:3007-3015 
123. Wilhelm EN et al (2014) Concurrent strength and endurance training exercise sequence does not affect neuromuscular adaptations in older men. Exp Gerontol 60:207-214

124. Fukumoto $\mathrm{Y}$ et al (2015) Age-related ultrasound changes in muscle quantity and quality in women. Ultrasound Med Biol 41(11):3013-3017

125. Schneider CA, Rasband WS, Eliceiri KW (2012) NIH Image to ImageJ: 25 years of image analysis. Nat Methods 9(7):671-675

126. Aagaard $P$ et al (2001) A mechanism for increased contractile strength of human pennate muscle in response to strength training: changes in muscle architecture. J Physiol 534(Pt. 2):613-623

127. Greening NJ et al (2015) Bedside assessment of quadriceps muscle by ultrasound after admission for acute exacerbations of chronic respiratory disease. Am J Respir Crit Care Med 192:810-816

128. Lieber RL, Friden J (2002) Morphologic and mechanical basis of delayed-onset muscle soreness. J Am Acad Orthop Surg 10(1):67-73

129. Austin N, Nilwik R, Herzog W (2010) In vivo operational fascicle lengths of vastus lateralis during sub-maximal and maximal cycling. J Biomech 43(12):2394-2399

130. Erskine RM et al (2009) In vivo specific tension of the human quadriceps femoris muscle. Eur J Appl Physiol 106(6):827-838

131. Korhonen MT et al (2009) Biomechanical and skeletal muscle determinants of maximum running speed with aging. Med Sci Sports Exerc 41:844-856

132. Cadore EL et al (2012) Echo intensity is associated with skeletal muscle power and cardiovascular performance in elderly men. Exp Gerontol 47:473-478

133. Wu R et al (2016) Effects of age and sex on neuromuscularmechanical determinants of muscle strength. Age 38:57

134. Muraki S, Fukumoto K, Fukuda O (2013) Prediction of the muscle strength by the muscle thickness and hardness using ultrasound muscle hardness meter. Springerplus 2:457

135. Varanoske AN et al (2017) Scanning plane comparison of ultrasound-derived morphological characteristics of the vastus lateralis. Clin Anat 30(4):533-542

136. Wilhelm EN et al (2014) Relationship between quadriceps femoris echo intensity, muscle power, and functional capacity of older men. Age 36:9625

137. Tang ZW et al (2015) Size of quadriceps femoris may contribute to thyrotoxic periodic paralysis. Med Hypotheses 85(6):749-753

138. Watson EL et al (2015) Progressive resistance exercise training in CKD: a feasibility study. Am J Kidney Dis 66(2):249-257

139. Kawai $\mathrm{H}$ et al (2018) Morphological and qualitative characteristics of the quadriceps muscle of communitydwelling older adults based on ultrasound imaging: classification using latent class analysis. Aging Clin Exp Res 30(4):283-291

140. Caresio C et al (2017) Fully automated muscle ultrasound analysis (MUSA): robust and accurate muscle thickness measurement. Ultrasound Med Biol 43(1):195-205

141. Welch D et al. (2018) Thigh muscle and subcutaneous tissue thickness measured using ultrasound imaging in older females living in extended care: a preliminary study. Aging Clin Exp Res 30(5):463-469

142. Shim DG, Kwon TY, Lee KB (2017) Rectus femoris muscle atrophy and recovery caused by preoperative pretibial traction in femoral shaft fractures-comparison between traction period. Orthop Traumatol Surg Res 103(5):691-695

143. Bemben MG (2002) Use of diagnostic ultrasound for assessing muscle size. J Strength Cond Res 16:103-108

144. Tok F et al (2011) Effects of botulinum toxin-A on the muscle architecture of stroke patients: an ultrasonographic study. J Rehabil Med 43(11):1016-1019
145. Tomlinson DJ et al (2014) The impact of obesity on skeletal muscle architecture in untrained young vs. old women. J Anat 225(6):675-684

146. Padhiar $\mathrm{N}$ et al (2008) Pennation angle of the soleus in patients with unilateral Achilles tendinopathy. Disabil Rehabil 30(20):1640-1645

147. Nagano Y, Higashihara A, Edama M (2015) Change in muscle thickness under contracting conditions following return to sports after a hamstring muscle strain injury-a pilot study. Asia Pac J Sports Med Arthrosc Rehabil Technol 2(2):63-67

148. Sjogaard G, Saltin B (1982) Extra- and intracellular water spaces in muscles of man at rest and with dynamic exercise. Am J Physiol 243(3):R271-R280

149. Thoirs K, English C (2009) Ultrasound measures of muscle thickness: intra-examiner reliability and influence of body position. Clin Physiol Funct Imaging 29(6):440-446

150. Berg HE, Tedner B, Tesch PA (1993) Changes in lower limb muscle cross-sectional area and tissue fluid volume after transition from standing to supine. Acta Physiol Scand 148(4):379-385

151. Cerniglia LM et al (2007) Effects of acute supine rest on midthigh cross-sectional area as measured by computed tomography. Clin Physiol Funct Imaging 27(4):249-253

152. Wagle JP et al (2017) Comparison of the relationship between lying and standing ultrasonography measures of muscle morphology with isometric and dynamic force production capabilities. Sports 5(4):88

153. Koda $\mathrm{H}$ et al (2018) Relationship between muscle strength asymmetry and body sway in older adults. J Aging Phys Act 26(3):457-461

154. Heckmatt JZ, Pier N, Dubowitz V (1988) Assessment of quadriceps femoris muscle atrophy and hypertrophy in neuromuscular disease in children. J Clin Ultrasound 16(3):177-181

155. Dupont AC et al (2001) Real-time sonography to estimate muscle thickness: comparison with MRI and CT. J Clin Ultrasound 29(4):230-236

156. Backhaus $\mathrm{M}$ et al (2001) Guidelines for musculoskeletal ultrasound in rheumatology. Ann Rheum Dis 60(7):641-649

157. Narici MV et al (1989) Changes in force, cross-sectional area and neural activation during strength training and detraining of the human quadriceps. Eur J Appl Physiol Occup Physiol 59(4):310-319

158. Kubo K et al (2003) Muscle architectural characteristics in young and elderly men and women. Int J Sports Med 24(2):125-130

159. Reeves ND, Narici MV, Maganaris CN (2004) In vivo human muscle structure and function: adaptations to resistance training in old age. Exp Physiol 89:675-689

160. Miljkovic I et al (2015) Greater skeletal muscle fat infiltration is associated with higher all-cause and cardiovascular mortality in older men. J Gerontol A Biol Sci Med Sci 70(9):1133-1140

161. Rollins KE et al (2016) The impact of sarcopenia and myosteatosis on outcomes of unresectable pancreatic cancer or distal cholangiocarcinoma. Clin Nutr 35(5):1103-1109

162. Montano-Loza AJ et al (2016) Sarcopenic obesity and myosteatosis are associated with higher mortality in patients with cirrhosis. J Cachexia Sarcopenia Muscle 7(2):126-135

163. Reimers K et al (1993) Skeletal muscle sonography: a correlative study of echogenicity and morphology. J Ultrasound Med 12(2):73-77

164. Ismail C et al (2015) Diagnostic ultrasound estimates of muscle mass and muscle quality discriminate between women with and without sarcopenia. Front Physiol 6:302

165. Pillen S et al (2009) Skeletal muscle ultrasound: correlation between fibrous tissue and echo intensity. Ultrasound Med Biol 35(3):443-446

166. Moher D et al (2009) Preferred reporting items for systematic reviews and meta-analyses: the PRISMA statement. J Clin Epidemiol 62(10):1006-1012 


\section{Affiliations}

\section{Stany Perkisas ${ }^{1,18}$ (D) . Stéphane Baudry ${ }^{2,18}$ • Jürgen Bauer ${ }^{3}$ - David Beckwée ${ }^{4,5}$ - Anne-Marie De Cock ${ }^{1}$. Hans Hobbelen ${ }^{6,19}$. Harriët Jager-Wittenaar ${ }^{6,20}$. Agnieszka Kasiukiewicz ${ }^{7}$. Francesco Landi ${ }^{8}$. Ester Marco ${ }^{9,10,11}$. Ana Merello ${ }^{12}$ - Karolina Piotrowicz ${ }^{13}$. Elisabet Sanchez ${ }^{12}$. Dolores Sanchez-Rodriguez ${ }^{11,14,15,16}$. Aldo Scafoglieri ${ }^{17,18} \cdot$ Alfonso Cruz-Jentoft ${ }^{12} \cdot$ Maurits Vandewoude $^{1,18}$}

1 University of Antwerp-University Geriatric Center, Leopoldstraat 26, 2000 Antwerp, Belgium

2 Laboratory of Applied Biology and Neurophysiologie, Université libre de Bruxelles, Brussels, Belgium

3 Department of Geriatric Medicine, Klinikum, Carl von Ossietzky University, Oldenburg, Germany

4 Rehabilitation Sciences Research Department, Vrije Universiteit Brussel, Brussels, Belgium

5 Department of Rehabilitation Sciences and Physiotherapy, Faculty of Medicine and Health Sciences, University of Antwerp, Antwerp, Belgium

6 Research Group Healthy Ageing, Allied Health Care and Nursing, Hanze University of Applied Sciences, Groningen, The Netherlands

7 Department of Geriatrics, Medical University in Bialystok, Białystok, Poland

8 Department of Geriatrics, Neuroscience and Orthopedics, Teaching Hospital “Agostino Gemelli”, Catholic University of the Sacred Heart School of Medicine, Rome, Italy

9 Physical and Rehabilitation Medicine, Parc de Salut Mar, Barcelona, Spain
10 Rehabilitation Research Group, Hospital del Mar Research Institute, Barcelona, Spain

11 Universitat Autònoma de Barcelona, Barcelona, Spain

12 University Hospital Ramón y Cajal, Madrid, Spain

13 Department of Internal Medicine and Gerontology, Faculty of Medicine, Jagiellonian University Medical College, Kraków, Poland

14 Geriatrics Department, Parc Salut Mar, Barcelona, Spain

15 Institut Hospital Del Mar d'Investigacions Mèdiques (IMIM), Barcelona, Spain

16 Universitat Pompeu Fabra, Barcelona, Spain

17 Faculty of Medicine and Pharmacy, Vrije Universiteit Brussel, Brussels, Belgium

18 Belgian Ageing Muscle Society, Liege, Belgium

19 Department of General Practice and Elderly Care Medicine, University of Groningen, University Medical Center Groningen, Groningen, The Netherlands

20 Department of Maxillofacial Surgery, University of Groningen, University Medical Center Groningen, Groningen, The Netherlands 